$\underset{\substack{\text { sonoms } \\ \text { conos }}}{\infty}$

${ }^{1}$ Department of Pediatrics/

Division of Pediatric Cardiology,

Mayo Clinic, Rochester,

Minnesota, USA; ${ }^{2}$ Department

of Medicine/Division of

Cardiovascular Diseases, Mayo

Clinic, Rochester, Minnesota,

USA; ${ }^{3}$ Department of Molecular

Pharmacology \& Experimental

Therapeutics, Mayo Clinic,

Rochester, Minnesota, USA

Correspondence to:

Dr M J Ackerman, Long OT

Syndrome Clinic and the Mayo

Clinic Windland Smith Rice

Sudden Death Genomics

Laboratory, Mayo Clinic,

Guggenheim 501, 200 First

Street SW, Rochester, MN

55905, USA; ackerman.

michael@mayo.edu

Accepted 6 July 2009

\title{
QTc: how long is too long?
}

\author{
J N Johnson, ${ }^{1}$ M J Ackermann ${ }^{1,2,3}$
}

\section{ABSTRACT}

Congenital long OT syndrome (LOTS) affects an estimated 1 in 2500 people and typically presents with syncope, seizures or sudden death. Whereas someone exhibiting marked prolongation of the OT interval with OTC exceeding 500 ms who was just externally defibrillated from torsades de pointes while swimming poses negligible diagnostic challenge as to the unequivocal probability of LOTS, the certainty is considerably less for the otherwise asymptomatic person who happens to host a QTc value coined "borderline" (QTC $\geqslant 440 \mathrm{~ms}$ ). Although a normal QT interval imparts a much lower risk of life-threatening events, it does not preclude a patient from nevertheless harbouring a potentially lethal LOTScausing genetic mutation. Indeed, genetic testing exerts significant diagnostic, prognostic and therapeutic implications. However, the 12-lead ECG remains the universal initial diagnostic test in the evaluation of LOTS and is subject to miscalculation, misinterpretation and mishandling. This review discusses the components of accurate QTc measurement and diagnosis, re-examines what is known about factors affecting OT interval measurement, and clarifies current recommendations regarding diagnosis of so-called "borderline" OT interval prolongation. The current guideline recommendations for the athlete with LOTS are also summarised.

Congenital long OT syndrome (LOTS) was first described clinically as Jervell and Lange-Nielsen syndrome and Romano Ward syndrome in the late 1950s and early 1960s. ${ }^{1-3}$ The trademark event for the symptomatic LOTS patient is the potentially lethal ventricular dysrhythmia called torsades de pointes. ${ }^{4}$ Torsades de pointes can precipitate syncope, seizures or sudden death, depending on whether the heart rhythm spontaneously reverts to normal rhythm or if the patient is defibrillated to normal rhythm before death occurs. ${ }^{56}$

LOTS affects an estimated 1 in 2500 people and is understood to be a collection of genetically distinct arrhythmogenic disorders resulting from genetic mutations in (primarily) cardiac potassium and sodium ion channels, termed "cardiac channelopathies". ? Mutations in proteins associated with ion channels but not part of the channels themselves can also cause LOTS. ${ }^{89}$ Overall, 12 LOTS-susceptibility genes have been discovered thus far. Mutations in the potassium channel genes KCNQ1 (LOT1) and KCNH2 (LOT2) as well as the sodium channel gene SCN5A (LOT3) account for $\sim 75 \%$ of all cases of clinically definite LOTS and comprise over $95 \%$ of genetically identifiable LOTS. $^{10-13}$

\section{DIAGNOSING LOTS: THE CHALLENGE}

The diagnosis of LOTS continues to be difficult. Some physicians continue to use a published clinical diagnostic assessment score (table 1) that incorporates personal and family history as well as ECG findings to compute "low", "intermediate" and "high" probabilities for the diagnosis of LOTS. ${ }^{14}$ Here, points are given for specific history components and findings on a 12-lead ECG, with a total score $\geqslant 4$ conveying a high probability of LOTS diagnosis. Points are awarded for OTc values exceeding $450 \mathrm{~ms}$ in males and $460 \mathrm{~ms}$ in females. A OTc value greater than $480 \mathrm{~ms}$ is worth 3 points. ${ }^{14}$

Unfortunately, this simple tool conceals the inherent difficulties with diagnosing this disease where, for example, the clinical presentation can masquerade as epilepsy. Further, a significant component of the score, namely the ECG index of cardiac repolarisation known as the QTc, can be computed erroneously by the ECG system or miscalculated by the physician performing his/her independent calculation. In fact, Viskin et $a l^{15}$ demonstrated that the QTc was calculated incorrectly (when compared with LOTS experts) by one-third of heart rhythm specialists and by nearly three-quarters of cardiologists.

Even when the correct OTc value has been obtained, its diagnostic significance can be misinterpreted, particularly when the value triggers the infamous designation of "borderline OT prolongation" (ie, OTc $\geqslant 440 \mathrm{~ms}$ ). At present, nearly half of the patients seen at our institution for a second opinion of a previously rendered diagnosis of LOTS are departing without such a diagnosis. ${ }^{16}$ Here, the majority of diagnostic reversals stem from miscalculated OTc values or the premature assignment of an LOTS diagnosis in the setting of low clinical probability scenarios (negative personal and family history) but with a "borderline" OTc. ${ }^{16}{ }^{17}$

Although the diagnosis may be relatively simple and readily apparent in a patient with a OTC persistently greater than $500 \mathrm{~ms}$ (especially if accompanied by typical LOTS-triggered cardiac events), primary care physicians and cardiologists are often confronted with patients diagnosed with "borderline" LOTS. These patients may or may not have an LQTS-specific history or complaint and have received an ECG for a variety of indications including screening pre-participation ECGs. The diagnosis of "borderline" OT prolongation or even "borderline LOTS" is commonly given when a patient has a OTc value between 440 and $470 \mathrm{~ms}{ }^{18}$ Within this range, however, there exists a significant "overlap zone", an area in which it is not possible to decipher whether or not a patient truly has LOTS simply on the basis of an ECG (fig 1). Indeed, fully $15 \%$ of the general population may have a OTc in the "borderline range". ${ }^{16}$ On the other hand, however, $25-35 \%$ of patients 
Table 1 LOTS Clinical Probability Score (Schwartz Score)

\begin{tabular}{|c|c|}
\hline Finding & Points \\
\hline \multicolumn{2}{|l|}{ History } \\
\hline \multicolumn{2}{|l|}{ Clinical history of syncope* } \\
\hline Without stress & 1 \\
\hline With stress & 2 \\
\hline Congenital deafness & 0.5 \\
\hline Family history of long OT syndrome $\dagger$ & 1 \\
\hline $\begin{array}{l}\text { Unexplained sudden death in a 1st degree family } \\
\text { member <age } 30^{\dagger}\end{array}$ & 0.5 \\
\hline \multicolumn{2}{|l|}{ ECG } \\
\hline \multicolumn{2}{|l|}{ Corrected OT interval (OTc by Bazett's formula) } \\
\hline $450 \mathrm{~ms}$ (in males) & 1 \\
\hline $460-470 \mathrm{~ms}$ & 2 \\
\hline$\geqslant 480 \mathrm{~ms}$ & 3 \\
\hline Torsade de pointes* & 2 \\
\hline T-wave alternans & 1 \\
\hline$\geqslant 3$ leads with notched $T$ waves & 1 \\
\hline Bradycardia ( $<2$ nd centile for age) & 0.5 \\
\hline
\end{tabular}

Adapted from Schwartz et al ${ }^{14}$ with permission from the American Heart Association, copyright 1993

$\leqslant 1=$ low probability; $1<$ score $<4=$ intermediate probability; $\geqslant 4=$ high probability.

${ }^{*}$ Syncope and torsade de pointes are mutually exclusive.

$\dagger$ Cannot count the same family member for both criteria.

harbouring an LQTS-causing mutation have a OTc $<440 \mathrm{~ms}$ and have been termed "silent carriers" or "concealed LQTS". ${ }^{19-21}$ From this significant overlap, it is easy to appreciate why LQTS can be both overdiagnosed and underdiagnosed.

\section{MEASUREMENT OF OTC: PITFALLS AND POINTERS}

As mentioned above, central to the assessment of a 12-lead ECG is the ability to accurately measure the OTc. Computer-derived measurements are fraught with errors, particularly in patients with complex $\mathrm{T}$-wave and U-wave arrangements. ${ }^{16}{ }^{22}$ As such, physicians-in-training should be taught that the computer's

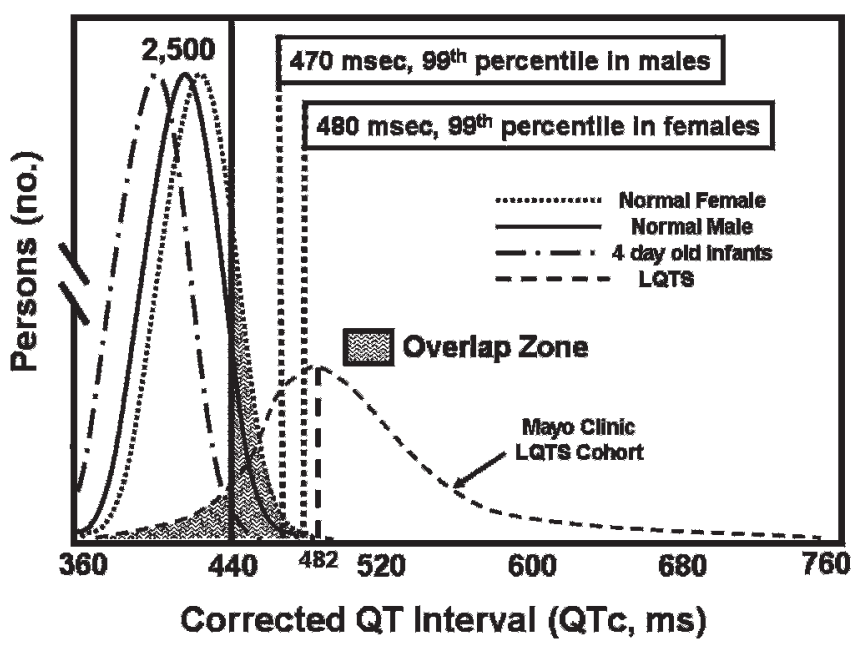

Figure 1 Distribution of QTc values for patients with and without long OT syndrome (LOTS). The "borderline" OTc level of $440 \mathrm{~ms}$ is shown with a solid line. Note the significant overlap between "normal" and OTc values of mutation-positive patients from Mayo's LOTS Clinic. Also note that the average OTc value in normal postpubertal females is on average $10 \mathrm{~ms}$ longer than that of normal postpubertal males. Modified from Taggart et $a /{ }^{16}$ with permission from the American Heart Association, copyright 2007.
QTc cannot be relied upon when a diagnosis of LQTS is in question and, must be verified manually. Complicating this recommendation, however, is the sobering observation from Viskin et $a l^{15}$ on the ability of cardiologists to accurately conduct this independent verification.

The QT interval is defined as the time duration between the onset of the ORS complex and the end of the $\mathrm{T}$ wave as it returns to baseline (fig 2), ideally measured using either lead II or lead V5 of the 12-lead ECG. There are no standards for interpreting prolonged OT intervals from Holter or 24/48 h ambulatory monitoring records; the "normal" OTc distribution at 2:30 am, for example, is not known, and thus QTc assessment by ambulatory monitoring is not recommended at present to invoke a diagnosis of LQTS. ${ }^{16}$ Regarding the 12-lead ECG, "normal" OTc values are generally considered to be between 350 and $440 \mathrm{~ms},{ }^{18}{ }^{23}$ but, as will be discussed in the next section, this consideration of OTc $>440 \mathrm{~ms}$ as indicative of "borderline OT prolongation" has probably been responsible for the greatest number of premature LOTS diagnostic renderings than the intrinsic fragility in the computation of the QTc in the first place.

Numerous methodologies for correcting OT intervals for heart rate have been proposed, ${ }^{24-26}$ but the most universally utilised method is that described by Bazett in $1920 .{ }^{27}$ Bazett calculated the OTc as the OT interval divided by the square root of the preceding RR interval. At normal heart rates, this formula is clinically useful, but begins to underestimate and overestimate the duration of cardiac repolarisation at extreme low and high heart rates, respectively. ${ }^{17}$ In the presence of sinus arrhythmia, the interpreting physician should calculate an average OTc from analysis of the entire 12-lead ECG rhythm strip. ${ }^{28}$ Although Martin et $a^{29}$ have proposed an alternative method in which the interpreter would apply Bazett's formula to the QT interval following the shortest available RR interval, this is strongly discouraged as it will result in a marked inflation/overestimation of the OTc and lead to overdiagnosis. In contrast, using only the longest $R R$ interval may underestimate the repolarisation index.

The $U$ wave is a common ECG finding, especially among adolescents, that is often a source of consternation for physicians reading ECGs. Most LQTS specialists recommend that a $U$ wave distinctly separate from and much smaller than the preceding $\mathrm{T}$ wave must be excluded from the OT interval measurement. ${ }^{23}$ Inclusion of such $U$ waves can easily "inflate"

\section{Bazett's Formula

$$
\mathrm{QTc}=\frac{\mathrm{QT}}{\sqrt{\mathrm{RR}}}
$$

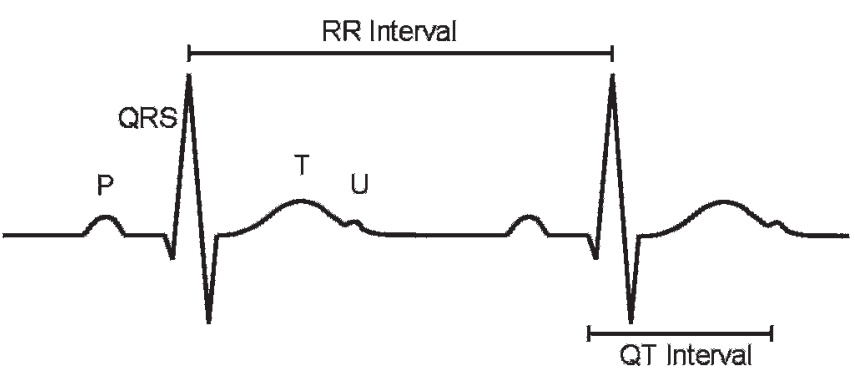

Figure 2 A diagrammatic representation of two cardiac cycles as recorded on an ECG. The RR and QT intervals are identified, along with a typical $U$ wave that should not be included in the QTc calculation. 
the QTc by 80-200 ms and unnecessarily precipitate a diagnosis of LOTS. ${ }^{16}$

To avoid mistakes in measurement of atypical $T$ waves, many specialists recommend a method where the end of the $T$ wave is considered to be the intersection of the tangent to the steepest slope of the last limb of the $\mathrm{T}$ wave and the baseline (fig 3).2. 30 This "teach-the-tangent", or as we refer to it "avoid-the-tail" method, was taught to a cohort of 151 medical students, who achieved higher accuracy of diagnosis (77\%) than previously described cohorts of non-cardiologists (21\%), cardiologists $(22 \%)$ and even electrophysiologists (62\%). ${ }^{15} 22$ Importantly, the accuracy of diagnosis was $96 \%$ for cardiologists specialising in LOTS. ${ }^{15} 22$

Finally, one must keep in mind that the QTc is not the only finding available on the 12-lead ECG. Specific T-wave and STsegment findings can give clues to the astute clinician as to the presence of pathological disease, particularly the presence of $\mathrm{T}$ wave alternans or diffuse $\mathrm{T}$-wave notching (fig 4). ${ }^{31}$ As such, the ECG must be examined critically for not only the length of the OT interval but also the look of the $\mathrm{T}$-wave and $\mathrm{T} / \mathrm{U}$-wave morphologies.

\section{CONFOUNDERS}

The QT interval is a fluid measurement that is influenced by the physiological and metabolic state of the patient at the time of the ECG. Owing to multiple variables interacting at any given time, patients may have different OT intervals during subsequent ECG examinations. The full extent of personal variability of the QT interval is currently unknown. For these reasons, use of specific OT interval cut-off values must be interpreted in the context of specific clinical information. For example, among the diagnostic reversals that we have encountered to date, $10 \%$ involved patients who had been referred after presenting to an emergency department with an episode of presyncope or syncope (typically vasovagal in description) where their first ever ECG demonstrated a "borderline" QT interval.

In addition, structural heart disease can play a significant role in altering the QT interval. ${ }^{18}$ Obtaining an exact measurement is particularly difficult in patients with left or right bundle branch block, found commonly in patients with a surgical history of congenital heart disease. ${ }^{32}$ Left ventricular hypertrophy may prolong the OT interval in the absence of known ion channel disease. $^{33}$ The presence of infarction or ischaemia in adults may also prolong the OT interval independently. ${ }^{34}$ Electrolyte abnormalities such as hypokalaemia, hypomagnesaemia and hypocalcaemia can lengthen the QT interval, whereas hypercalcaemia may

\section{"Teach The Tangent"}

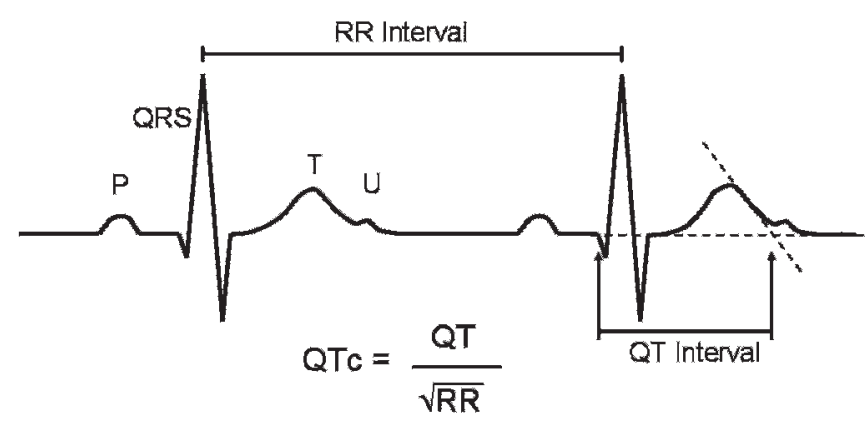

Figure 3 A repeat diagrammatic representation of two cardiac cycles as recorded on an ECG, with demonstration of the "teach-the-tangent" method of QTc measurement as described by Postema et al. ${ }^{22}$

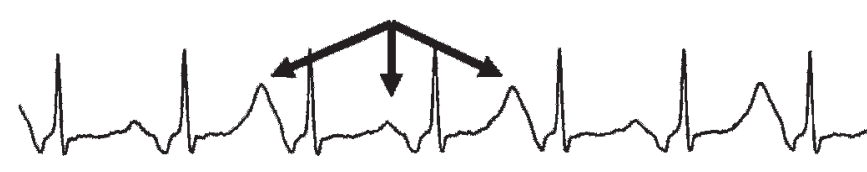

Figure 4 An ECG rhythm strip demonstrating significant macroscopic T-wave alternans. Note the alternating T-wave morphology and vector.

shorten the QT interval..$^{35}$ Prolonged OT intervals have been found in patients with diabetic ketoacidosis and are associated with the level of ketosis, even in the absence of electrolyte abnormalities. ${ }^{36}$ Patients with anorexia nervosa have longer QT intervals than controls with normal body weight. ${ }^{37}$ Finally, the OTc of normal subjects has been shown to demonstrate diurnal variation (longer during sleep and during REM sleep in particular), ${ }^{38}{ }^{39}$ and the OTC in otherwise healthy men is significantly longer in winter months than summer months. ${ }^{40}$

Numerous drugs have been well documented to prolong the QT interval as an unwanted side effect. ${ }^{41}$ The presence of these drugs must be ruled out during the workup for LOTS, and they should be avoided as a rule when a diagnosis of LOTS is considered. An up-to-date list of these drugs is available at http://www.qtdrugs.org.

\section{OT INTERVALS IN ATHLETES}

The prevalence of QT interval prolongation in asymptomatic elite athletes was recently reported as $0.4 \%$, or 1 in 286 patients. ${ }^{30}$ Of the positive patients in this study, only the three with QT intervals over $500 \mathrm{~ms}$ had either a positive provocation test or positive genetic test, and were thus excluded from participating in their sport. Four other patients with QTc values between 460 and $500 \mathrm{~ms}$ were studied further, but not disqualified from their sport because of negative family histories and negative provocative testing. A 2006 publication on the Italian pre-participation screening programme reported disqualification of $2 \%$ of all athletes on the basis of screening, with only five athletes out of 42386 ultimately diagnosed with LQTS. ${ }^{42}$ The majority of disqualifications in the Italian cohort were for cardiomyopathy, hypertension, valvular disease and non-LQTS rhythm abnormalities. ${ }^{42}$ In each of these studies, the European guideline cut-off values for diagnosing prolonged OT were used: $440 \mathrm{~ms}$ for males and $460 \mathrm{~ms}$ for females. ${ }^{43}$

The 36th Bethesda Conference guidelines were published in 2005 and established recommendations for athletic activity restrictions in patients with LQTS. ${ }^{44}$ A near-all-encompassing competitive sports disqualification was advised for any patient with a history of out-of-hospital cardiac arrest or LOTS-related syncopal episode, regardless of underlying genotype or QTc. For asymptomatic patients, a QTc of $\geqslant 470 \mathrm{~ms}$ in males or $\geqslant 480 \mathrm{~ms}$ in females was the recommended limitation to only class IA sports of billiards, bowling, cricket, curling, golf and riflery, with further liberalisation possible if the asymptomatic patient had the LQT3 genotype. A loosening of the competitive sports disqualifications was deemed reasonable for asymptomatic genotype-positive LOTS patients with OTc values in the overlapping/borderline range, except for competitive swimming in athletes with concealed LQT1. ${ }^{44}$

The European Society of Cardiology (ESC) in 2006 also published a set of athletic activity guidelines for patients with LQTS. ${ }^{45}$ Patients were recommended to be completely restricted from participating in competitive sports if they were symptomatic, had a prolonged OT interval (over 440-470 ms in men, over 460-480 $\mathrm{ms}$ in women), or were carriers of a known 
genetic mutation (regardless of their QTc). Patients considered at low risk of sudden death were allowed to participate in light to moderate leisure activity. Any sports involving sudden bursts of activity or genotype-specific triggers were absolutely contraindicated in patients with LQTS. Again, in contrast with the Bethesda guidelines, the recommendation for competitive sports restriction was unchanged for genotype-positive patients with normal or borderline QT values. ${ }^{45}$

As is evident, there are profound implications of hosting QTc values that are awarded 1, 2 or 3 points in the LQTS diagnostic score card and consequently convey "intermediate" probability for LQTS (table 1). However, what truly is the predictive utility of say a QTc of $450 \mathrm{~ms}$ in an otherwise healthy man or a QTc of $470 \mathrm{~ms}$, or dare we say, $480 \mathrm{~ms}$ or even $490 \mathrm{~ms}$, in a healthy woman? As we shall see, clinical context and pretest probability mean everything.

\section{HOW LONG IS TOO LONG?}

As seen in fig 1, there is substantial overlap in the distribution of OTc between otherwise healthy subjects and patients with genetically confirmed LOTS. Among patients seen with genetically proven LQTS at the Mayo Clinic, the mean (SD) QTc is 482 (50) $\mathrm{ms}$ and, at present, ranges from $365 \mathrm{~ms}$ as the lowest value in a patient with genetically definite LOT1 to a high of $800 \mathrm{~ms}$. Consistent with the published literature, $40 \%$ of our genotyped LOTS population exhibit QTc values $<460 \mathrm{~ms}$. However, this prevalence of concealed or normal QT intervalLQTS must be juxtaposed carefully with the normal distribution of QTc values, including the prevalence of normal people who simply exhibit "upper limit of normal" OTc values. In other words, there are a lot more normal people with high normal, upper limit of normal, borderline QT prolongation than there are people with a 1 in 2500 condition known as LQTS where half of them have a OTc $<480 \mathrm{~ms}$. This important subtlety appears to have been overlooked given the current mini-epidemic of overdiagnosed LQTS. ${ }^{16}$ In fact, when the original LOTS diagnostic criteria were published in 1985, a diagnostic cut-off QTc value of $440 \mathrm{~ms}$ was used, regardless of patient age or gender. ${ }^{46}$ The criteria were updated in 1993 to now award 1 point to a male with a QTc of $450 \mathrm{~ms}$ and 2 points to both males and females when a OTc of $460 \mathrm{~ms}$ is reached. ${ }^{14}$ This gender differentiation was added after adult women were found to have longer OTc values than men by $\sim 10 \mathrm{~ms}^{47}$ Nevertheless, any person with a OTc $\geqslant 460 \mathrm{~ms}$ receives 2 points in the LQTS diagnostic score card, which is translated as "intermediate probability" for LOTS. As we will see, this clinical assignment of "intermediate probability" is only valid if one accepts a $2 \%$ chance of LOTS as indicative of "intermediate probability".

Several recent reviews have proposed an "upper limit" of $460 \mathrm{~ms}$ in patients $<15$ years of age, $470 \mathrm{~ms}$ for adult females, and 450 ms for adult males. ${ }^{17}{ }^{23}$ In this algorithm, any QTc value within $20 \mathrm{~ms}$ of these designated upper limits is considered "borderline". ${ }^{23}$ Consequently, an adult male with a OTc of $431 \mathrm{~ms}$ is considered by these criteria to exhibit "borderline" QT prolongation. Others have proposed that any patient with a QTc between 440 and 470 ms be labelled "borderline."18 A 2005 European protocol proposed the use of a OTc value greater than $440 \mathrm{~ms}$ in males and $460 \mathrm{~ms}$ in females as a definition of a "prolonged" OTc. ${ }^{30}{ }^{43}$ Even the latest 2009 AHA/ACCF/HRS Recommendations for the Standardization and Interpretation of the Electrocardiogram states that a OTc $\geqslant 450 \mathrm{~ms}$ (males) and $\geqslant 460 \mathrm{~ms}$ (females) "be considered a prolonged QT interval". ${ }^{48}$

With respect to "borderline", although the literature cites the "normal range" as being 350-440 ms, it is often not recognised that $10-20 \%$ of otherwise healthy postpubertal individuals have QTc values beyond this so-called "normal range" (fig 1). In

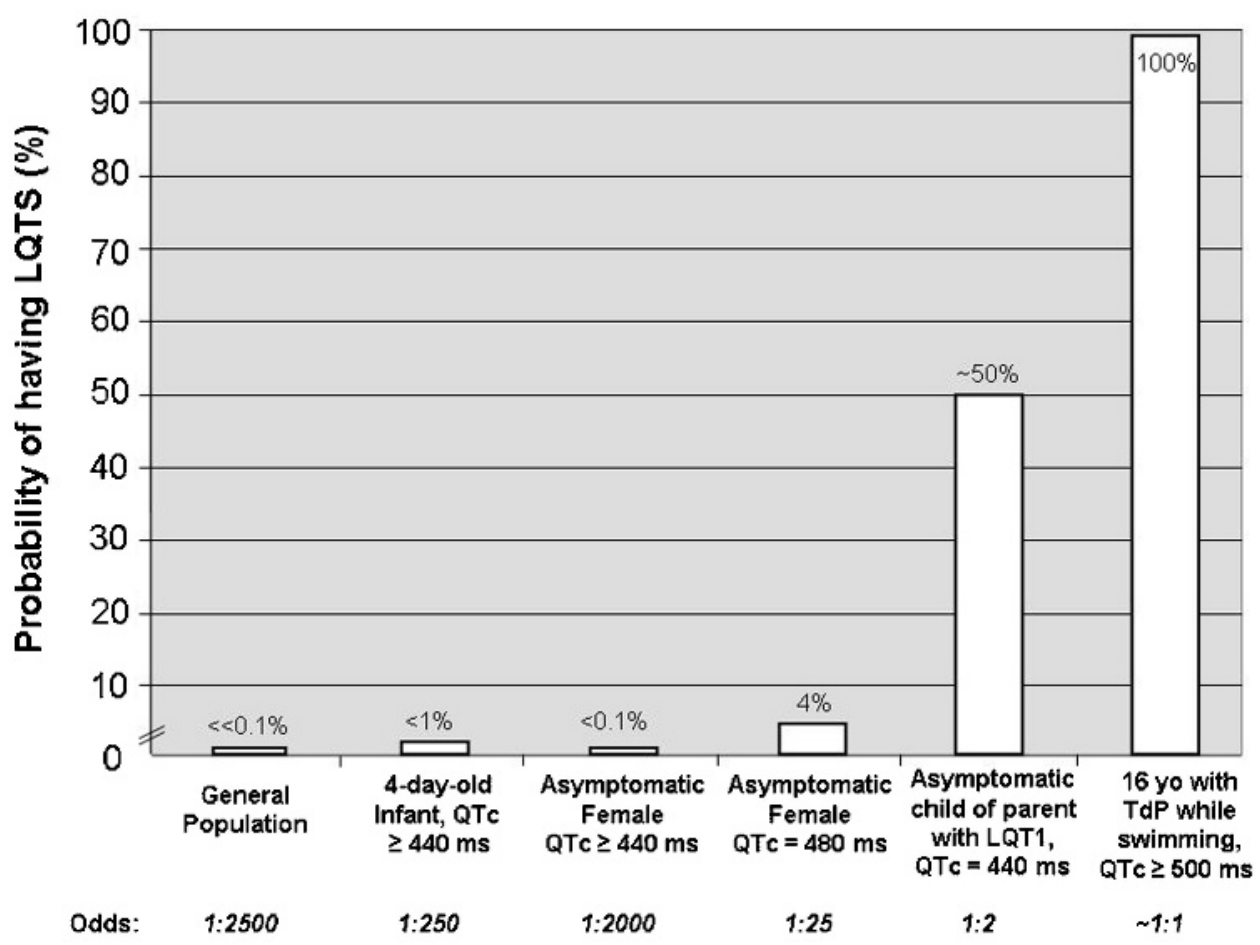

Figure 5 Effect of clinical setting on the relative probability of having long OT syndrome (LOTS). Note the relatively low predictive value of a OTC $>440 \mathrm{~ms}$ in any asymptomatic patient compared with the patient with personal symptoms or a family history suggestive of LQTS. TdP, torsades de pointes. 
1. If an ECG is ordered to "rule out" LOTS, the computerderived OTc must be manually verified. If the manually derived OT interval is within $10 \mathrm{~ms}$ of the computer's OT interval by either the "tangential" or "avoid-the-tail" method, one can be confident that the computer knows how to compute the average RR interval, use the heart rate correction formula, and generate an accurate QTc.

2. All manually measured QTc values must be interpreted in the context of the patient's personal and family history.

3. A "borderline" QTc value is not sufficient evidence for a diagnosis of LQTS or even borderline/possible LOTS.

4. In a patient with a personal or family history suspicious for LOTS (exercise-induced syncope/seizures for example), further work-up for LOTS including treadmill exercise stress testing and/or epinephrine OT stress testing, and genetic testing should be considered even if the OTc is "borderline" or possibly even normal. In other words, the story should drive the depth and aggressiveness of the evaluation not the 12-lead ECG. However, exercise-induced syncope in the context of a QTc $<460 \mathrm{~ms}$ is far more likely to stem from catecholaminergic polymorphic ventricular tachycardia than from concealed type 1 LOTS.

5. As a potentially lethal, highly treatable condition affecting 1 in 2500 people, LOTS certainly warrants consideration of not only pre-participation athlete screening but also universal screening. From a screening perspective, a QTc value $\geqslant 470 \mathrm{~ms}$ for a $2-4$-week-old infant screening programme or a $0 T c \geqslant 500 \mathrm{~ms}$ in adolescents/adults may represent far more optimal "penalty box" screening values with an estimated positive predictive value $>50 \%$. Such a screen would produce far fewer subsequent evaluations needed to distinguish the true positives from the false positives while capturing the subpopulation of LOTS most at risk of a future LQTS-precipitated cardiac event.

Furthermore, subsequent genetic testing of these positively screened index cases would identify their LOTS-

susceptibility mutation over $75 \%$ of the time, thereby permitting genetic testing of appropriate family members, which would enable a large proportion of the subpopulation of lower risk, normal QT interval/borderline OT interval/ concealed LOTS to be essentially "back-filled."

other words, $10-20 \%$ of the human population has "borderline QT prolongation" per any of the aforementioned definitions, guidelines or cut-offs. As shown in fig 1, 0.5-1\% of men and women in the adult human population receive at least 2 points and a clinical diagnostic score indicative of "intermediate probability" LOTS.

Figure 5 depicts the critical importance of context in the determination of when "long is too long". There would be little debate as to the near- $100 \%$ pretest probability of LQTS for someone with documented torsades de pointes while swimming and a QTc of $500 \mathrm{~ms}$. On the other hand, there would be tremendous debate as to the probability of LOTS in an asymptomatic, postpubertal female with a OTc of $481 \mathrm{~ms}$. By the LQTS diagnostic score card, 3 points and "intermediate probability" would be assigned. By the ESC 2006 position statement, she would be disqualified from all sports regardless of symptomatic state. However, if such a OTc value represents the 99-99.5th centile among women, this stand-alone value

\section{What is already known on this topic}

- Long OT syndrome (LOTS) is a potentially lethal yet highly treatable genetic heart rhythm disorder ("cardiac channelopathy") that affects an estimated 1 in 2500 people.

- Investigators have searched for simple non-invasive methods to aid in the diagnostic elucidation and risk stratification of patients with suspected LOTS.

- Despite significant advances in our understanding of its pathogenic underpinnings, genotype-phenotype relationships and therapeutic strategies, LOTS still remains both underdiagnosed and overdiagnosed.

\section{What this study adds}

- Whereas many authors have attempted to pick a specific "cutoff" OTc value for athletic disqualification and risk stratification, this study emphasises that all QTC measurements should be interpreted in the context of the patient's personal and family history.

- More importantly, the age-dependent and sex-dependent normal distribution of this particular index of repolarisation (OTc) in both health and disease must be kept in sharp focus.

confers a positive predictive value for LOTS of $<5 \%$. If such a QTc cut-off value $(480 \mathrm{~ms})$ were adopted as part of a universal screen for females (470 ms for males), 20-25 people could be prematurely and incorrectly diagnosed with LQTS for every single person where this screening ECG exposed LOTS in an otherwise asymptomatic host. Again, everything is context dependent, as these same cut-off values applied to universal screening of infants instead of postpubertal adults might have positive predictive values approaching $50 \% .{ }^{49} 50$

Now consider the $440 \mathrm{~ms}$ threshold that is still held in some circles as worthy of receiving "borderline OT prolongation", which unfortunately is almost always incorrectly and quickly translated as "borderline LQTS". As shown in figs 1 and 5, this value is achieved or exceeded by $2.5 \%$ of 4 -day-old infants ${ }^{51}$ and by $10-20 \%$ of postpubertal adults. Accordingly, an adult woman with no personal or family history suggestive of LOTS has $<0.1 \%$ probability of LOTS simply because her screening ECG registered a QTc of $443 \mathrm{~ms}$. In sharp contrast, an equally asymptomatic teenage athlete with the same OTc who is the offspring of a mother with A341V-KCNQ1-mediated LQT1 has a $50 \%$ chance (not $<0.1 \%$ chance) of inheriting her mother's LQT1-susceptibility mutation and may have LQTS.

\section{CONCLUSIONS}

Primary care physicians and cardiologists are often confronted with patients who exhibit "upper limit of normal" or "borderline QT prolongation" and then give the diagnosis of "possible/ borderline" LQTS. The extreme overlap in this index of cardiac repolarisation between patients with genetically established LOTS and otherwise healthy subjects emphasises the critical importance of considering the whole clinical picture and the various diagnostic modalities besides the 12-lead ECG to distinguish the person who has a highly treatable, potentially life-threatening condition known as LQTS from the one whose QTc is just "borderline" just because. 
Acknowledgements: MA's research programme was supported by the Mayo Clinic Windland Smith Rice Comprehensive Sudden Cardiac Death Program, the Dr Scholl Foundation, the CJ Foundation for SIDS, the Hannah Wernke Memorial Foundation, an Established Investigator Award from the American Heart Association, and the National Institutes of Health (HD42569).

Competing interests: MA is a consultant for PGxHealth, Medtronic, Pfizer, Mayo Clinic Health Solutions, the Mayo Clinic Windland Smith Rice Sudden Death Genomics Laboratory and receives royalties from LOTS and CPVT genetic testing (FAMILION) by PGxHealth.

Provenance and peer review: Commissioned; not externally peer reviewed.

\section{REFERENCES}

1. Jervell A, Lange-Nielsen F. Congenital deaf-mutism, functional heart disease with prolongation of the Q-T interval and sudden death. Am Heart J 1957;54:59-68.

2. Romano C, Gemme G, Pongiglione R. [Rare cardiac arrythmias of the pediatric age. li. Syncopal attacks due to paroxysmal ventricular fibrillation. (Presentation of $1 \mathrm{st}$ case in Italian pediatric literature)]. (In Italian.) Clin Pediatr (Bologna) 1963;45:656-83.

3. Ward 0C. A New familial cardiac syndrome in children. J Ir Med Assoc 1964;54:103-6.

4. Ackerman MJ. Cardiac channelopathies: it's in the genes. Nat Med 2004;10:463-4.

5. Moss AJ. Long QT syndrome. JAMA 2003;289:2041-4.

6. Vincent GM, Timothy K, Fox J, et al. The inherited long QT syndrome: from ion channel to bedside. Cardiol Rev 1999;7:44-55.

7. Ackerman MJ. The long QT syndrome: ion channel diseases of the heart. Mayo Clin Proc 1998;73:250-69.

8. Mohler PJ, Schott JJ, Gramolini A0, et al. Ankyrin-B mutation causes type 4 longOT cardiac arrhythmia and sudden cardiac death. Nature 2003:421:634-9.

9. Vatta M, Ackerman MJ, Ye B, et al. Mutant caveolin-3 induces persistent late sodium current and is associated with long-OT syndrome. Circulation 2006:114:2104-12

10. Tester DJ, Will ML, Haglund CM, et al. Effect of clinical phenotype on yield of long QT syndrome genetic testing. J Am Coll Cardiol 2006;47:764-8.

11. Chen L, Marquardt ML, Tester DJ, et al. Mutation of an A-kinase-anchoring protein causes long-QT syndrome. Proc Natl Acad Sci USA 2007;104:20990-5.

12. Medeiros-Domingo A, Kaku T, Tester DJ, et al. SCN4B-encoded sodium channel beta4 subunit in congenital long-QT syndrome. Circulation 2007;116:134-42.

13. Ueda K, Valdivia C, Medeiros-Domingo A, et al. Syntrophin mutation associated with long OT syndrome through activation of the nNOS-SCN5A macromolecular complex. Proc Natl Acad Sci USA 2008;105:9355-60.

14. Schwartz PJ, Moss AJ, Vincent GM, et al. Diagnostic criteria for the long QT syndrome. An update. Circulation 1993;88:782-4.

15. Viskin S, Rosovski U, Sands AJ, et al. Inaccurate electrocardiographic interpretation of long QT: the majority of physicians cannot recognize a long QT when they see one. Heart Rhythm 2005;2:569-74.

16. Taggart NW, Haglund CM, Tester DJ, et al. Diagnostic miscues in congenital longOT syndrome. Circulation 2007:115:2613-20.

17. Vetter VL. Clues or miscues? How to make the right interpretation and correctly diagnose long-OT syndrome. Circulation 2007:115:2595-8.

18. Levine E, Rosero SZ, Budzikowski AS, et al. Congenital long OT syndrome: considerations for primary care physicians. Cleve Clin J Med 2008;75:591-600.

19. Vincent GM, Timothy KW, Leppert M, et al. The spectrum of symptoms and OT intervals in carriers of the gene for the long-0T syndrome. $N$ Engl J Med 1992:327:846-52.

20. Vyas H, Hejlik J, Ackerman MJ. Epinephrine QT stress testing in the evaluation of congenital long-QT syndrome: diagnostic accuracy of the paradoxical QT response. Circulation 2006:113:1385-92.

21. Priori SG, Napolitano C, Schwartz PJ. Low penetrance in the long-OT syndrome: clinical impact. Circulation 1999:99:529-33.

22. Postema PG, De Jong JS, Van der Bilt IA, et al. Accurate electrocardiographic assessment of the OT interval: teach the tangent. Heart Rhythm 2008;5:1015-18.

23. Goldenberg I, Moss AJ, Zareba W. OT interval: how to measure it and what is "normal". J Cardiovasc Electrophysiol 2006;17:333-6.
24. Davey P. A new physiological method for heart rate correction of the $\mathrm{QT}$ interval. Heart 1999;82:183-6.

25. Sagie A, Larson MG, Goldberg RJ, et al. An improved method for adjusting the QT interval for heart rate (the Framingham Heart Study). Am J Cardiol 1992;70:797-801.

26. Fridericia L. The duration of systole in the electrocardiogram of normal subjects and of patients with heart disease. Acta Med Scand 1920:469-86

27. Bazett H. An analysis of the time-relations of electrocardiograms. Heart 1920:353-370.

28. Vincent GM, Richard J. Calculation of the OTc interval during sinus arrhythmia in patients suspected to have long OT syndrome. Circulation 2001;104:II-690-1.

29. Martin AB, Perry JC, Robinson JL, et al. Calculation of QTC duration and variability in the presence of sinus arrhythmia. Am J Cardiol 1995;75:950-2.

30. Basavarajaiah S, Wilson $\mathrm{M}$, Whyte $\mathrm{G}$, et al. Prevalence and significance of an isolated long OT interval in elite athletes. Eur Heart J 2007;28:2944-9.

31. Moss AJ, Zareba W, Benhorin J, et al. ECG T-wave patterns in genetically distinct forms of the hereditary long OT syndrome. Circulation 1995:92:2929-34.

32. Talbot S. QT interval in right and left bundle-branch block. Br Heart J 1973;35:288-91.

33. Jouven $\mathbf{X}$, Hagege $A$, Charron $P$, et al. Relation between OT duration and maximal wall thickness in familial hypertrophic cardiomyopathy. Heart 2002;88:153-7.

34. Kenigsberg DN, Khanal S, Kowalski M, et al. Prolongation of the OTC interval is seen uniformly during early transmural ischemia. J Am Coll Cardiol 2007:49:1299-305.

35. Diercks DB, Shumaik GM, Harrigan RA, et al. Electrocardiographic manifestations: electrolyte abnormalities. J Emerg Med 2004;27:153-60.

36. Kuppermann N, Park J, Glatter K, et al. Prolonged QT interval corrected for heart rate during diabetic ketoacidosis in children. Arch Pediatr Adolesc Med 2008;162:544-9.

37. Lesinskiene $\mathbf{S}$, Barkus A, Ranceva $\mathrm{N}$, et al. A meta-analysis of heart rate and OT interval alteration in anorexia nervosa. World J Biol Psychiatry 2008;9:86-91.

38. Browne KF, Prystowsky E, Heger JJ, et al. Prolongation of the Q-T interval in man during sleep. Am J Cardiol 1983:52:55-9.

39. Lanfranchi PA, Shamsuzzaman AS, Ackerman MJ, et al. Sex-selective QT prolongation during rapid eye movement sleep. Circulation 2002:106:1488-92.

40. Beyerbach DM, Kovacs RJ, Dmitrienko AA, et al. Heart rate-corrected QT interval in men increases during winter months. Heart Rhythm 2007;4:277-81.

41. Napolitano C, Bloise R, Priori SG. Long QT syndrome and short QT syndrome: how to make correct diagnosis and what about eligibility for sports activity. J Cardiovasc Med (Hagerstown) 2006; 7:250-6.

42. Corrado D, Basso C. Pavei A, et al. Trends in sudden cardiovascular death in young competitive athletes after implementation of a preparticipation screening program. JAMA 2006;296:1593-601.

43. Corrado D, Pelliccia A, Bjornstad HH, et al. Cardiovascular pre-participation screening of young competitive athletes for prevention of sudden death: proposal for a common European protocol. Consensus Statement of the Study Group of Sport Cardiology of the Working Group of Cardiac Rehabilitation and Exercise Physiology and the Working Group of Myocardial and Pericardial Diseases of the European Society of Cardiology. Eur Heart J 2005;26:516-24.

44. Zipes DP, Ackerman MJ, Estes NA 3rd, et al. Task Force 7: arrhythmias. J Am Coll Cardiol 2005; 45:1354-63.

45. Heidbuchel $\mathbf{H}$, Corrado D, Biffi A et al. Recommendations for participation in leisuretime physical activity and competitive sports of patients with arrhythmias and potentially arrhythmogenic conditions. Part II: ventricular arrhythmias, channelopathies and implantable defibrillators. Eur J Cardiovasc Prev Rehabil 2006;13:676-86.

46. Schwartz PJ. Idiopathic long OT syndrome: progress and questions. Am Heart J 1985; 109:399-411.

47. Merri M, Benhorin J, Alberti M, et al. Electrocardiographic quantitation of ventricular repolarization. Circulation 1989:80:1301-8.

48. Rautaharju PM, Surawicz B, Gettes LS. AHA/ACCF/HRS recommendations for the standardization and interpretation of the electrocardiogram. Part IV: the ST segment, $\mathrm{T}$ and U waves, and the OT interval. JACC Cardiovasc Imaging 2009;53:982-91.

49. Quaglini S, Rognoni C, Spazzolini C, et al. Cost-effectiveness of neonatal ECG screening for the long QT syndrome. Eur Heart J 2006;27:1824-32.

50. Schwartz PJ, Quaglini S. Cost-effectiveness of neonatal ECG screening for the long OT syndrome: reply. Eur Heart $J$ 2007;28:137.

51. Schwartz PJ, Stramba-Badiale M, Segantini A, et al. Prolongation of the OT interva and the sudden infant death syndrome. N Engl J Med 1998:338:1709-14. 